indications for ordering a TTE and used BSE Guidance to classify the indications as appropriate, inappropriate, or unclear.

Results Thirty-two requests for TTE were received over the study period. The median patient age was 74 years [range 32-93 years]. Just over half of the patients 17/32 (52\%) were male. The most common indications for TTE were to evaluate valvular pathology 11/32 (34\%); either initial assessment 5/32 $(16 \%)$ or surveillance of known valvular disease 6/32 (18\%). The second most common indication was to assess cardiomyopathy $8 / 32$ (25\%), followed by evaluation of arrhythmias, palpitations or syncope 4/32 (13\%). When compared with BSE Guidance, 18 (56\%) requests were appropriate and 11 (34\%) were inappropriate. Three (9\%) requests were deemed unclear due to the lack of information contained in the request.

Conclusion An almost equal number of females and males were referred for TTE. The median age reflects the older cohort of patients at our hospital. Valvular pathology; either for diagnostic assessment or repeat evaluation was the most common indication for echocardiography, More than onethird of TTE requests received were not clinically indicated and combined with unclear requests, this amounted to more than $40 \%$ of requests. We would recommend implementation of BSE Guidance at our hospital to provide guidance for physicians making requests and ensure appropriate utilisation of limited resources for clinically indicated echocardiograms.

\section{NEOINTIMAL TISSUE COVERAGE AND CHARACTERIZATION ASSESSMENT USING OPTICAL COHERENCE TOMOGRAPHY SURVEILLANCE SIX MONTHS AFTER IMPLANTATION OF BIORESORBABLE SCAFFOLDS VERSUS CONVENTIONAL EVEROLIMUS ELUTING STENTS IN ISAR-ABSORB MI TRIAL}

\begin{abstract}
${ }^{1} \mathrm{H}$ Rai, ${ }^{2} \mathrm{~F}$ Alfonso, ${ }^{3} \mathrm{M}$ Maeng, ${ }^{4} \mathrm{C}$ Bradaric, ${ }^{1} \mathrm{~J}$ Wiebe, ${ }^{2} \mathrm{~J}$ Cuesta, ${ }^{3} \mathrm{E}-\mathrm{H}$ Christiansen, ${ }^{1} \mathrm{~F}$ Harzer, ${ }^{1} \mathrm{~S}$ Cassese, ${ }^{4} \mathrm{P}$ Hoppmann, ${ }^{1} \mathrm{R}$ Colleran, ${ }^{4} \mathrm{~S}$ Schneider, ${ }^{4,5} \mathrm{~K}-\mathrm{L}$ Laugwitz, ${ }^{1,5} \mathrm{~A}$ Kastrati, ${ }^{1,5} \mathrm{RA}$ Byrne. ${ }^{1}$ Deutsches Herzzentrum München, Technische Universität München, Munich, Germany; ${ }^{2}$ Hospital Universitario de La Princesa Madrid, Madrid, Spain; ${ }^{3}$ Aarhus University Hospital, Aarhus, Denmark; ${ }^{4}$ Klinikum rechts der Isar, Technische Universität München, Munich, Germany; ${ }^{5}$ DZHK (German Centre for Cardiovascular Research), partner site Munich Heart Alliance, Munich, Germany
\end{abstract}

\subsection{6/heartjnl-2020-ICS.51}

Background Bioresorbable scaffolds (BRS) were introduced to overcome the limitations of permanent metallic stent implantation. Optical coherence tomography (OCT) can provide important insights on vessel wall healing at follow-up. We compared OCT-assessed healing at 6 months after implantation of everolimus-eluting BRS and everolimus-eluting metallic stents (EES) in patients treated for acute myocardial infarction (AMI).

Methods ISAR-Absorb MI is a multicentre, 2:1 randomized trial comparing outcomes of patients with AMI stented with BRS or conventional EES. Angiographic surveillance was planned for all patients at 6-8 months follow-up; surveillance with OCT at follow-up was discretionary. For the present analysis, patients with OCT follow-up were included and images analyzed at a core laboratory. Tissue characterization using grey-scale signal intensity (GSI) analysis was done for neointimal regions of interest (ROI) with thickness of 100 to $400 \mu \mathrm{m}$. ROI's were classified as mature using a cut-off GSI score of 109.7. Generalized linear mixed model was used as appropriate.

Results 70 patients in the BRS arm and 32 patients in the EES arm had OCT available for analysis at a median followup of 216 days. Minimum lumen area [5.13 (3.95, 6.70) vs. $\left.4.93(3.84,6.99) \mathrm{mm}^{2}\right]$ and minimum stent area $[5.78(4.88$, $7.34)$ vs. $6.39(4.77,7.45) \mathrm{mm}^{2}$ ] were comparable between BRS and EES. In total 2,237 frames with 19,827 struts were assessed. Overall strut coverage was better with BRS compared to $\operatorname{EES}(97.5 \%$ vs. $90.9 \%, \mathrm{p}<0.001)$, while malapposed struts $(1.1 \%$ vs. $0.5 \%, \mathrm{p}=0.51)$ were more common with EES. Neointimal coverage was comparable in both stent groups [85.5 (61.9, 124.1) vs. $69.5(32.7,127.5) \mu \mathrm{m}$ in BRS and EES groups, $\mathrm{p}=0.20]$. GSI analysis in 95 cases showed that immature ROIs were numerically more common in the EES group as compared to the BRS group (75.4 vs. $57.0 \%$; $\mathrm{p}=0.35$ ).

Conclusions In selected patients undergoing OCT imaging at 6-8 months after implantation of BRS and conventional EES for AMI, we observed generally favorable healing characteristics with high rates of strut coverage and fewer areas of immature neointimal areas with BRS in comparison to EES.

\section{OUT OF HOSPITAL CARDIAC ARRESTS IN YOUNG ADULTS (16-35 YEARS); A SIX-YEAR REVIEW OF THE IRISH OUT-OF-HOSPITAL CARDIAC ARREST REGISTER}

${ }^{1} \mathrm{R}$ Tanner, ${ }^{2} \mathrm{~S}$ Materson, ${ }^{3} \mathrm{~J}$ Galvin, ${ }^{1} \mathrm{C}$ Deasy. ${ }^{1}$ Cork University Hospital, Cork, Ireland; ${ }^{2}$ National Ambulance Service Lead, Clinical Strategy and Evaluation, National Ambulance Service, HSE, Ireland; ${ }^{3}$ Mater Misericordiae University Hospital, Dublin, Ireland

\subsection{6/heartjnl-2020-ICS.52}

Introduction Out-of-hospital cardiac arrests (OHCA) in the young population have only been examined in a limited number of regional studies. Hence, we sought to describe OHCA characteristics and predictors of survival to hospital discharge for young Irish adults.

Methods An observational analysis of the national Irish out-ofhospital cardiac arrest register for all OHCAs aged 16 to $\leq 35$ years between January 2012 and December 2017 was performed. Multivariable logistic regression was used to determine the independent predictors of survival to hospital discharge.

Results A total of 1,005 OHCAs aged 16 to $\leq 35$ years (24.3\% female, median age 27 years [IQR 23-32]) had resuscitation attempted over the study period. OHCA location was most commonly in the home $(61.3 \%, \mathrm{n}=616)$ and a minority of OHCAs occurred in areas of sport or recreation $3.5 \%$, $\mathrm{n}=35)$. A non-medical aetiology was most prevalent $(59 \%$, $\mathrm{n}=593$ ) with asphyxiation, trauma and drug overdoses

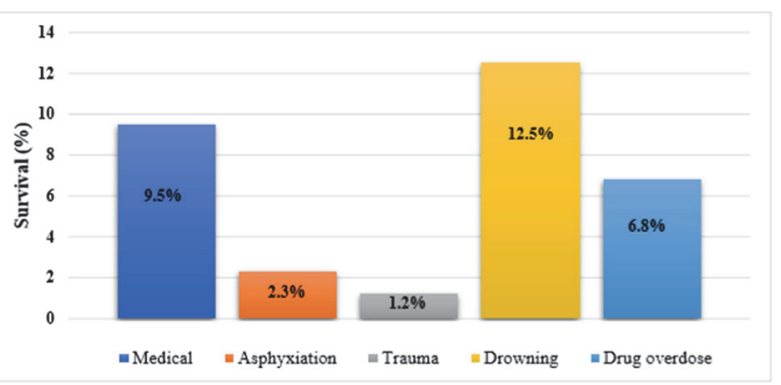

Abstract 52 Figure 1 Survivial to hospital discharge based on OHCA aetiology $(n=1,005)$ 
accounting for over $90 \%$ of these cases. Two-thirds of patients received bystander $\mathrm{CPR}$ and a defibrillator was attached to the patient in $14.9 \%(n=150)$ of OHCAs before arrival of emergency medical services with a shock being ultimately delivered in 3.9\% $(n=39)$ of cases. Overall survival to hospital discharge for the cohort was $6.0 \%(n=60)$ and no significant change in survival was noted over the 6-year study period. The rate of survival was dependent on OHCA aetiology, (figure 1). Independent predictors of survival to hospital discharge included bystander witnessed OHCA, a shockable initial rhythm and a bystander defibrillation attempt.

Conclusions The high prevalence of non-medical OHCAs and the OHCA location need to be considered when developing OHCA care pathways and preventative strategies to reduce the burden of OHCAs in young adults.

\section{HYPERTENSION IN THE ACUTE MEDICAL ASSESSMENT UNIT IN ST. LUKE'S GENERAL HOSPITAL KILKENNY - ARE WE ADHERING TO GUIDELINES?}

K Millar, C Crowley, P Cotter. St Luke's Hospital, Kilkenny, Ireland

10.1136/heartjnl-2020-ICS.53

Background Hypertension is a common reason for GP referral to the Acute Medical Assessment Unit (AMAU) in St. Luke's Hospital, Kilkenny (SLK). Hypertensive emergency requires urgent and aggressive blood pressure control to limit endorgan damage. Management of hypertension has been proven to reduce the incidence of cardiac events, cardiovascular death and stroke. The aims of this audit were: 1. To ascertain what percentage of patients referred with hypertension were diagnosed with hypertensive emergency. 2. To review whether or not patients referred with hypertension had had appropriate work up (with electrocardiogram and urine dipstick.) 3. To review what percentage of patients deemed not to have hypertensive emergency received unindicated stat doses of antihypertensives. 4. To assess what percentage of patients were managed in compliance with the ESC-ESH Hypertension Guidelines 2018 on discharge.

Methods We carried out a retrospective review of the case notes and discharge letters of 106 patients who were referred to the AMAU by GPs with hypertension between January 2019 and January 2020.

Results 91 patients were included in the study. 35 (38\%) were male and $56(62 \%)$ were female. The median age of patients was 60.2 patients $(2.2 \%)$ were diagnosed with hypertensive emergency. Both were managed with oral antihypertensives. 83 patients (91\%) were asked about symptoms of hypertension, 86 patients (95\%) had an ECG. Only 26 (29\%) had a urine dipstick. Of the 89 patients deemed not to have hypertensive emergency, $43(48 \%)$ received a stat dose of an oral antihypertensive. 11 patients $(12 \%)$ had their stage of hypertension documented. Of these, 8 (73\%) were discharged on an appropriate antihypertensives. 24 patients $(27 \%)$ had stage 1 hypertension, $27(30 \%)$ had stage 2 and 38 (43\%) had stage 3. On discharge, 40 patients (45\%) were discharged on dual/triple antihypertensive regimens (in accordance with ESC-ESH guidelines.)

Of the 89 patients discharged from the AMAU, 69 (78\%) were booked for a 24 hour ABPM and 26 (29\%) were referred for TTE.
Conclusion This audit highlights numerous areas for improvement in the management of patients presenting to the AMAU in SLK with hypertension. It revealed that almost half of patients deemed to have no evidence of malignant hypertension/end-organ damage requiring immediate blood pressure control received at least one stat dose of an anti-hypertensive. The staging and management of hypertension remains a confusing area for some NCHDs, evidenced by the fact that only $12 \%$ of patients had their hypertension staged during their review. Positively, almost $80 \%$ of patients who presented to the AMAU with hypertension were booked for an outpatient 24 hour ABPM, however only 29\% were booked for a TTE. We aim to develop a proforma for managing patients presenting with hypertension and re-educate our staff on the investigation and management of hypertensive patients in order to closer align our practices with ESC-ESH guidelines.

\section{RESIDUAL RISK IN CARDIAC REHAB: CAN WE REDUCE- IT MORE? ELIGIBILITY FOR ICOSAPENT ETHYL IN PATIENTS ATTENDING CARDIAC REHABILITATION}

${ }^{1} \mathrm{~S}$ Gaine, ${ }^{2} \mathrm{JJ}$ Coughlan, ${ }^{3} \mathrm{~V}$ Maher, ${ }^{3} \mathrm{M}$ Waters. 'St James's Hospital, Dublin, Ireland; ${ }^{2}$ German Heart Centre, Munich, Germany; ${ }^{3}$ Tallaght University Hospital, Tallaght, Dublin, Ireland

\subsection{6/heartjnl-2020-ICS.54}

Introduction Patients with elevated triglycerides (TG) are at increased risk for ischemic events despite statin therapy and controlled low-density lipoprotein cholesterol (LDL-C). The REDUCE IT trial showed that a highly purified Eicosapentaenoic acid (EPA) ester, Icosapent Ethyl or Ethyl Eicosapentaenoic acid (E-EPA), reduces the risk of ischemic events and cardiovascular (CV) death in patients with elevated TG levels despite statin therapy. The NNT for the first occurrence of major adverse cardiac events for the five-point primary composite endpoint was 21. E-EPA is also a dominant strategy from a cost-effectiveness perspective in the study. Thus, the 2019 ESC/EAS guidelines recommend E-EPA for patients with persistently raised TGs despite treatment with a statin. Our aim was to assess the proportion of patients attending cardiac rehabilitation who may benefit from E-EPA therapy as per REDUCE-IT trial criteria and the 2019 ESC/EAS guidelines.

Methods We prospectively collected data on all cardiac rehabilitation patients in our centre in 2018/2019. We then performed a hierarchal analysis of these patients to determine the percentage of patients post MI/CABG that would meet criteria for E-EPA as per REDUCE-IT trial criteria and ESC/EAS guidelines. $=$

The REDUCE IT trial criteria were:

- $\geq 45$ years and established ASCVD

- 50 years with DM and at least one other CV RF

- Fasting TG level of 1.69 to $5.63 \mathrm{mmol} / \mathrm{L}$

- LDL-C level of 1.06 to $2.59 \mathrm{mmol} / \mathrm{L}$

- Receiving a stable dose of a statin for at least 4 weeks

The initial trial protocol enrolled patients with a TG level as low as $1.52 \mathrm{mmol} / \mathrm{L}$ to account for the $\sim 10 \%$ variability in TG levels. This protocol was amended and changed the lower level for TG to $2.26 \mathrm{mmol} / \mathrm{L}$. We analysed patients as per both protocols. 